Research Article

\title{
Establishment and Validation of a Liquid Chromatography-Tandem Mass Spectrometry Method for the Determination of Tigecycline in Critically Ill Patients
}

\author{
Fen Yao, ${ }^{1}$ Yifan Wang, ${ }^{1}$ Yating Hou $\mathbb{D}^{2},{ }^{2}$ Xipei Wang, ${ }^{3}$ Jinhua Lan, ${ }^{4}$ Zheng Wu, ${ }^{4}$ \\ Yirong Wang, ${ }^{4}$ and Chunbo Chen $\mathbb{1}^{1,5,6}$ \\ ${ }^{1}$ School of Biology and Biological Engineering, South China University of Technology, Guangzhou 510006, Guangdong, China \\ ${ }^{2}$ Department of Emergency Medicine, Maoming People's Hospital, 101 Weimin Road, Maoming 525000, Guangdong, China \\ ${ }^{3}$ Department of Medical Sciences, Guangdong Provincial People's Hospital, Guangdong Academy of Medical Sciences, \\ Guangdong Cardiovascular Institute, 106 Zhongshan Er Road, Guangzhou 510080, China \\ ${ }^{4}$ Department of Critical Care Medicine, Guangdong Provincial People's Hospital, Guangdong Academy of Medical Sciences, \\ 106 Zhongshan Er Road, Guangzhou 510080, Guangdong, China \\ ${ }^{5}$ Department of Intensive Care Unit of Cardiovascular Surgery, Guangdong Cardiovascular Institute, \\ Guangdong Provincial People's Hospital, Guangdong Academy of Medical Sciences, \\ Laboratory of South China Structural Heart Disease, 96 Dongchuan Road, Guangzhou 510080, Guangdong, China \\ ${ }^{6}$ The Second School of Clinical Medicine, Southern Medical University, Guangzhou, China
}

Correspondence should be addressed to Chunbo Chen; gghccm@163.com

Received 25 October 2020; Accepted 3 December 2020; Published 29 December 2020

Academic Editor: Chanbasha Basheer

Copyright $(2020$ Fen Yao et al. This is an open access article distributed under the Creative Commons Attribution License, which permits unrestricted use, distribution, and reproduction in any medium, provided the original work is properly cited.

Utilizing tigecycline-d9 as an internal standard (IS), we establish and validate a simple, effective, and rapid liquid chromatography-tandem mass spectrometry (LC-MS/MS) method for the quantitative measurement of tigecycline (TGC) in patient plasma. Acetonitrile was used as a precipitant to process plasma samples by a protein precipitation method. The analyte and IS were separated on an HSS T3 $(2.1 \times 100 \mathrm{~mm}, 3.5 \mu \mathrm{m})$ chromatographic column using isocratic program with a mobile phase comprising of $80 \%$ solvent A (water containing $0.1 \%$ formic acid (v/v) with $5 \mathrm{mM}$ ammonium acetate) and $20 \%$ solvent B (acetonitrile) with a flow rate of $0.3 \mathrm{~mL} / \mathrm{min}$. The mass spectrometer, scanning in multireaction monitoring (MRM) mode and using an electrospray ion source (ESI), operated in the positive-ion mode. The ion pairs used for quantitative analysis were $m / z$ $586.4 \longrightarrow 513.3$ and $\mathrm{m} / z 595.5 \longrightarrow 514.3$ for TGC and the IS, respectively. The range of the linear calibration curve obtained with this approach was $50-5000 \mathrm{ng} / \mathrm{ml}$. Intra- and interbatch precision for TGC quantitation were less than $7.2 \%$, and the accuracy ranged from 93.4 to $101.8 \%$. The IS-normalized matrix effect was 87 to $104 \%$. Due to its high precision and accuracy, this novel method allows for fast quantitation of TGC with a total analysis time of $2 \mathrm{~min}$. This approach was effectively applied to study the pharmacokinetics of TGC in critically ill adult patients.

\section{Introduction}

Tigecycline (TGC) is the first member of the glycylcycline class of antimicrobial agents and is associated with refractory infections in critically ill patients $[1,2]$. Though high-dose tigecycline ( $200 \mathrm{mg}$ loading dose, $100 \mathrm{mg} \mathrm{q} 12 \mathrm{~h}$ ) were recommended for the treatment of severe infections [3-5], a black box warning of increased all-cause mortality was issued by the Food and Drug Administration (FDA) [6]. The area under the concentration-time curve from 0 to $24 \mathrm{~h}$ at stead -state $\left(\mathrm{AUC}_{24}\right)$ divided by the minimal inhibitory concentration (MIC) is often used as the pharmacokinetic/ pharmacodynamic (PK/PD) index, with target values calculated for various infections [7-11]. Body surface area and creatinine clearance were reported to have a significant influence on clearance (CL) $[10,12,13]$. Renal impairment 
frequently occurs in critically ill patients [14-18]. Therefore, therapeutic drug monitoring (TDM) of TGC is essential for the optimal use of TGC in clinical practice.

Determinations of TGC in patient plasma by liquid chromatography with ultraviolet (UV) or tandem mass spectrometry (LC-MS/MS) methods were reported previously [19-22]. However, long run times, time-consuming sample preparation, and large sample volumes are needed in these methods [10, 11, 13, 21]. Jiao Xie described an LC-MS/ MS method with a short run time of 5 min but a relatively large sample volume of $200 \mu \mathrm{L}$ [22]. A small sample volume was used in Rong Shao's LC-MS/MS method, but a concentration step was still needed after protein precipitation [19, 23-25]. In addition, hemolysis may occur during blood collection and processing, and hyperlipidemic samples are not rare in clinical sampling, especially in critically ill patients. The presence of hemolyzed or hyperlipidemic plasma samples may affect the analyte recovery efficiency [26-29]. In addition, the matrix effect of TGC in such abnormal plasma has not yet been reported.

The current research established a sensitive, simple, and rapid LC-MS/MS method for the quantitative analysis of TGC in patient plasma, utilizing tigecycline-d9 (TGC-d9) as an internal standard (IS). The influence of abnormal plasma on the matrix effect of TGC was investigated in the full method validation. This approach was applied to analyze 222 samples collected from 74 ICU patients for a population pharmacokinetic (PPK) study.

\section{Materials and Methods}

2.1. Chemicals and Reagents. Tigecycline (Lot: L290P45, 99\% purity) was obtained from Beijing Bailingwei Technology Company. [t-Butyl-d9]-tigecycline (internal standard, IS, Lot: 0532724 , 95\% purity) was purchased from Cayman Chemical Company. High-pressure liquid chromatography(HPLC-) grade formic acid was acquired from SigmaAldrich (St. Louis, MO, USA), and HPLC-grade acetonitrile was bought from Thermo Fisher Scientific (Waltham, MA, USA). Preparation of the ultrapure water was accomplished through a Milli-Q Plus water purification system.

2.2. Instrumentation. The HPLC system comprises of an LC20AB pump, a CTO-20A column oven, and an SIL-20AC/ HT autosampler (Shimadzu Corporation, Japan) coupled with a 4000Qtrap triple quadrupole mass spectrometer with a heated electrospray ionization source (Applied Biosystems, Foster City, CA, USA). Collection and processing of data was accomplished with Analyst 1.4.2 software.

\subsection{LC-MS/MS Conditions}

2.3.1. Chromatographic Conditions. On an HSS T3 column $(2.1 \times 100 \mathrm{~mm}, 3.5 \mu \mathrm{m}$ particles) (Waters Company), the analyte and IS were separated by isocratic elution at a flow rate of $0.3 \mathrm{~mL} / \mathrm{min}$. The mobile phase was composed of water consisting of $0.1 \%$ formic acid (v/v) with $5 \mathrm{mM}$ ammonium acetate $(80 \% \mathrm{~A})$ and acetonitrile $(20 \% \mathrm{~B})$. The needle rinsing solution of the autosampler was methanol: ultrapure water $(\mathrm{v} / \mathrm{v}, 70: 30)$. The column oven was set at $30^{\circ} \mathrm{C}$, and the total analysis time was only $2 \mathrm{~min}$. The sample volume injected by the autosampler was $3 \mu \mathrm{L}$.

2.3.2. Mass Spectrometric Conditions. The mass spectrometer, scanning in multireaction monitoring (MRM) mode and using an electrospray ion source (ESI), operated in positive ion mode. The ion pairs used for quantitative analysis were $m / z 586.4 \longrightarrow 513.3$ for TGC (Figure $1(\mathrm{a})$ ) and $\mathrm{m} / \mathrm{z} 595.5 \longrightarrow 514.3$ for the IS (Figure $1(\mathrm{~b})$ ). The positive electrospray ionization source (ESI+) temperature was $550^{\circ} \mathrm{C}$; the ion spray voltage was $4500 \mathrm{~V}$; and the declustering potential, entrance potential, and collision energies were $52 \mathrm{eV}, 10 \mathrm{eV}$, and $20 \mathrm{eV}$ for TGC and $70 \mathrm{eV}, 10 \mathrm{eV}$, and $42 \mathrm{eV}$ for the IS, respectively.

2.4. Preparation of Stock and Working Solutions. To prepare a tigecycline stock solution with a mass concentration of $1 \mathrm{mg} /$ $\mathrm{ml}, 20.02 \mathrm{mg}$ of tigecycline standard compound was weighed into a brown volumetric flask and diluted with $20 \mathrm{ml}$ of ultrapure water. Calibration curves were obtained for the working solutions of tigecycline diluted with ultrapure water to obtain concentrations of 1000, 2000, 4000, 10000, 20000, 40000,80000 , and $100000 \mathrm{ng} / \mathrm{ml}$. One milliliter of ultrapure water was added to the $0.5 \mathrm{mg}$ internal standard to make an internal standard stock solution with a mass concentration of $500 \mu \mathrm{g} / \mathrm{ml}$. Five milliliters of water was added to $10 \mu \mathrm{l}$ of the internal standard stock solution to prepare a $1000 \mathrm{ng} / \mathrm{ml}$ internal standard working solution. All the stock working solutions were stored in a refrigerator at $-80^{\circ} \mathrm{C}$ and thawed and mixed at room temperature before use.

2.5. Preparation of Standard and Quality Control Samples. Standard curves and quality control (QC) samples were prepared as follows: $10 \mu \mathrm{L}$ of each working solution was added to $190 \mu \mathrm{L}$ of blank plasma to prepare standard samples with final concentrations of 50,100,200,500, 1000, 2000, 4000 , and $5000 \mathrm{ng} / \mathrm{mL}$. A similar method was used to acquire QC samples with plasma concentrations of $150,750,2250$, and $3800 \mathrm{ng} / \mathrm{ml}$. These samples were kept at $-80^{\circ} \mathrm{C}$ until use.

2.6. Sample Preparation. Patient plasma samples were thawed at room temperature. To precipitate the protein in the plasma samples, a $50 \mu \mathrm{l}$ aliquot of the plasma samples was added to a $1.5 \mathrm{~mL}$ Eppendorf tube, mixed with a $20 \mu \mathrm{l}$ IS working solution aliquot, and vortexed for 20 seconds. Subsequently, $200 \mu \mathrm{l}$ of acetonitrile was added into the solution, followed by vigorous mixing for 1 minute and centrifugation at $12000 \mathrm{rpm}$ at $4^{\circ} \mathrm{C}$ for 15 minutes. Three microliters of the supernatant was injected into the HPLC system.

2.7. Method Validation. Validation of the method was consistent with the guidance of the Fourth Edition of the Chinese Pharmacopoeia 2015, 9012 "Guidelines for 


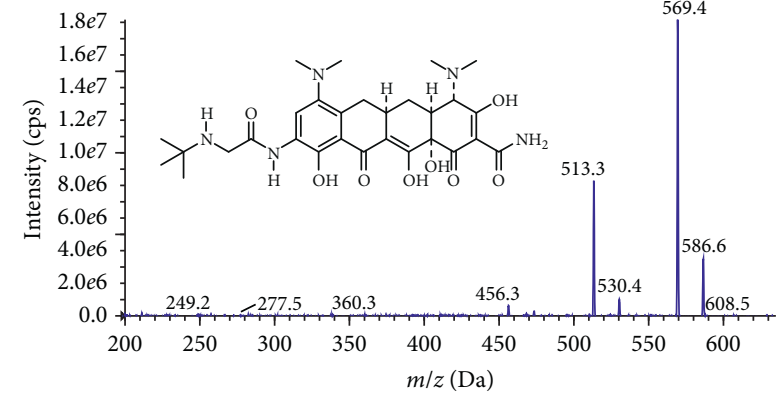

(a)

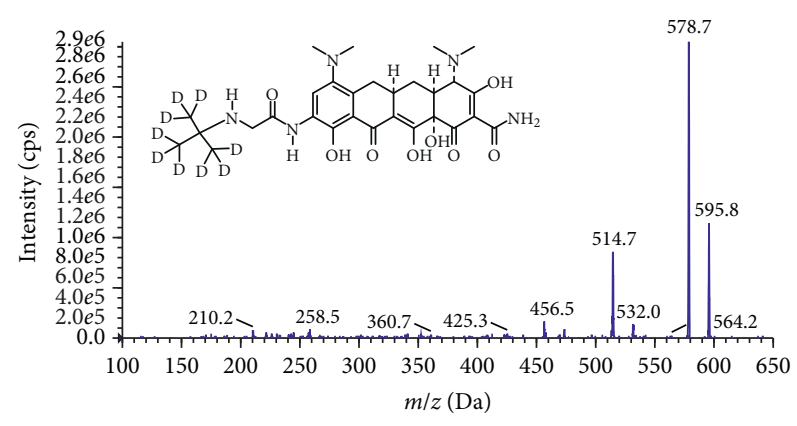

(b)

Figure 1: Chemical structures and product ion mass spectra of the $[\mathrm{M}+\mathrm{H}]^{+}$ions of (a) tigecycline and (b) [t-butyl-d9]-tigecycline (IS).

Validation of Quantitative Analysis Methods for Biological Samples," including the selectivity, carryover effects, lower limit of quantification (LLOQ), linearity, accuracy and precision, matrix effect, and stability.

2.7.1. Selectivity and Carryover Effect. At least, six blank plasma samples collected from different individuals should be analyzed to test the selectivity of the method. The interference effect of each blank sample was determined by examining the peak area of retention times of TGC and the IS. When the response of the interfering component was less than $20 \%$ of the LLOQ and $5 \%$ of the internal standard, there was no interference under the verification criteria.

According to carryover criteria, the carryover effects should be evaluated by injecting blank samples after the upper limit of quantification (ULOQ) sample. Carryover in blank samples after the ULOQ sample should not exceed $20 \%$ of the LLOQ and should not exceed $5 \%$ of the internal standard.

2.7.2. LLOQ and Linearity. LLOQ is the lowest concentration of analyte in a sample that can be reliably quantified with receivable accuracy and precision. Eight calibration standards were researched over a calibration range of $50-5000 \mathrm{ng} / \mathrm{mL}$ TGC in plasma to assess the linearity of the measurement. Each calibration curve was composed of eight calibration concentration levels and a double blank sample, a zero sample. A zero sample is a blank sample with only internal standard added. Linearity was determined by fitting the peak area ratio to the analyte concentration using weighted least squares analysis and a weighting factor of $1 / x^{2}$. In method validation, at least, 3 calibration curves should be evaluated. The back-calculated concentration of the calibration standard should generally be within $\pm 15 \%$ of the labeled value, and the LLOQ should be within $\pm 20 \%$. At least, $75 \%$ calibration standards, containing, at least, 6 effective concentrations, should meet the abovementioned standards. If the result of a calibration standard sample does not meet these standards, the standard sample should be rejected. The calibration curve that does not contain this standard sample should be re-evaluated.
2.7.3. Accuracy and Precision. Interbatch and intrabatch accuracy and precision were assessed by evaluating six replicate QC samples at LLOQ, low QC (LQC1), low-medium QC (LQC2), medium QC (MQC), and high QC (HQC)concentrations $(50,150,750,2250$, and $3800 \mathrm{ng} / \mathrm{mL}$ ) in, at least, three batches performed over, at least, two days. The accuracy calculated as the percent deviation from the nominal concentration describes how close the measured value is to the nominal concentration of the analyte. Precision is determined by the coefficient variation (CV\%) of the calculated vs. nominal concentration. The average accuracy should generally be within $\pm 15 \%$ of the labeled value of the quality control sample, and the accuracy of the LLOQ should be within $\pm 20 \%$ of the labeled value. For quality control samples, CV\% between batches and within batches should generally not be more than $15 \%$, and the coefficient of variation obtained for the LLOQ should not exceed $20 \%$.

2.7.4. Matrix Effect. Since the accuracy of the LC-MS/MS experiment might be affected by matrix effects, we then investigated the matrix effects by examining the blank matrix collected from six individuals at three QC levels (150, 750 , and $3800 \mathrm{ng} / \mathrm{mL}$ ). The matrix factor of an analyte and IS should be computed by determining the ratio of the peak area with the matrix (quantified by adding the analyte and IS after blank matrix extraction) to the corresponding peak area short of the matrix (the pure solution of the analyte and IS). The IS-normalized matrix factor (MF) was obtained by determining the ratios of the MF values of the analyte and the IS. The CV\% of the IS-normalized MF computed from six batches of the matrix should not be greater than $15 \%$.

In addition to the normal matrix effect, attention should also be paid to the matrix effects of other samples, such as hemolyzed samples and hyperlipidemic plasma samples. To investigate the effect of the hemolysis and hyperlipidemic status on quantitative detection, simulated hemolytic plasma containing $2 \%$ lysed blood ( $2 \%$ hemolytic plasma) was prepared by adding $20 \mu \mathrm{L}$ of whole blood subjected to multiple freeze-thaw cycles to $980 \mu \mathrm{L}$ of normal blank plasma. Hyperlipidemic plasma was mimicked by adding $15 \mu \mathrm{L}$ of a $20 \%$ medium-/long-chain fatty acid emulsion to $985 \mu \mathrm{L}$ of normal blank plasma to obtain a final triglyceride content of approximately $1.7 \mathrm{mM}$. 
2.7.5. Stability. Using three replicate samples with low and high QC levels, we assessed the stability of TGC in plasma under various storage and processing conditions. We evaluated the stability of TGC in human plasma in the shorttime by incubating QC samples at room temperature and storing at $2-8^{\circ} \mathrm{C}$ for 24 hours. The long-term stability of QC samples stored at $-80^{\circ} \mathrm{C}$ for 130 days was determined. For evaluation of postpreparation, QC samples were collected before injection and placed in the autosampler for $30 \mathrm{~h}$ at $30^{\circ} \mathrm{C}$. The freeze-thaw stability was determining after five complete freeze-thaw cycles $\left(-80^{\circ} \mathrm{C}\right.$ to room temperature) were performed. If the measured value is within the acceptable accuracy $( \pm 15 \%)$ and CV\% $(\leq 15 \%)$ range, these samples are considered stable.

2.8. Application to the PPK Study. The verified method was effectively applied to a PPK study of 74 patients in two ICU datasets. The study was approved by the Research Ethics Committee of Guangdong Provincial People's Hospital (Guangdong Academy of Medical Science) (approval no. GDREC2018268H (R1)). Three plasma samples were collected from each patient after the blood concentration reached steady state, usually after the fifth dose. The collection times were at the end of the installation, 4-6 hours after intravenous administration, and before the next dose. All blood samples were collected into EDTA-K2 tubes and immediately centrifuged at 3,000 rpm for $10 \mathrm{~min}$; the resultant plasma samples were stored and frozen at $-80^{\circ} \mathrm{C}$ until analysis. A total of 222 plasma samples were collected and analyzed.

\section{Results and Discussion}

3.1. Method Optimization. The mass spectrometer, scanning in multireaction monitoring (MRM) mode and using an electrospray ion source (ESI), operated in positive ion mode. The ion pairs used for quantitative analysis were $\mathrm{m} / \mathrm{z}$ $586.4 \longrightarrow 513.3$ and $m / z 595.5 \longrightarrow 514.3$ for TGC and the IS, respectively (Figure 1). TGC is sensitive to the $\mathrm{pH}$ of the mobile phase; therefore, to obtain better chromatograms, a buffer containing $0.1 \%$ formic acid (v/v) with $5 \mathrm{mM}$ ammonium acetate was used as mobile phase $\mathrm{A}$. The total run time was only $2 \mathrm{~min}$, which is much shorter than the run times of previously published methods [19, 22].

\subsection{Method Validation}

3.2.1. Selectivity and Carryover Effect. The retention times of TGC and the IS were $0.95 \mathrm{~min}$ and $0.94 \mathrm{~min}$, respectively. As indicated, the interference peaks of endogenous substances observed at the retention time of TGC and the IS were negligible (Figure 2), which implies that the MRM chromatograms are typical of (a) double-blank plasma, (b) blank plasma spiked only with IS, (c) TGC (LLOQ) and IS spiked with blank plasma, and (d) plasma from a patient. This confirmed the specificity of the developed method for the quantitative analysis of TGC in human plasma.
By adding a double blank sample after the ULOQ sample, the observed peak area is less than $20 \%$ of the LLOQ and $5 \%$ of the IS, indicating that there is no carry over of the analyte and IS existed in the method.

3.2.2. The LLOQ and Linearity. LLOQ is $50 \mathrm{ng} / \mathrm{mL}$. A typical linear regression equation is $y=15.7 x+0.0498(r=0.9978)$, where $y$ represents the ratios of the TGC peak area to the IS peak area and $x$ stands for the concentrations of TGC. The peak area ratios and concentration showed a linear relationship in the range of $50-5000 \mathrm{ng} / \mathrm{mL}$, with correlation coefficients $>0.99$. The slopes and correlation coefficients were consistent between batches. A linear range of 50-5000 ng/mL was adequate for the PK estimates of TGC in ICU patients receiving standard or high-dose regimens because almost all TGC concentrations were in this range. A higher ULOQ was needed for the application of a high-dose regimen, in which concentrations above $3000 \mathrm{ng} / \mathrm{mL}$ might be observed in the elimination phase of TGC [4].

3.2.3. Accuracy and Precision. The intraday precision (CV\%) ranged from 1.7 to $3.6 \%$ over the five concentration levels of QC samples, and the accuracy was within 93.8 to $101.8 \%$. For the interday experiments, the precision varied from 3.7 to $7.2 \%$, and the accuracy was within 93.4 to $99.7 \%$ at these levels. The results of accuracy and precision analyses are summarized in Table 1. A low LLOQ needed additional sample volume or a concentration process after protein precipitation, as reported $[19,22]$, but no concentration lower than the LLOQ was found in the present study. An extremely low LLOQ was unnecessary in the TDM of TGC.

3.2.4. Matrix Effect. The CV\% of the IS-normalized matrix effect was within the range from $4 \%$ to $8.5 \%$, which is in accordance with the guidelines (Table 2). More importantly, we found that the IS-normalized matrix effect of TGC in the hemolytic and hyperlipidemic plasma was similar to that in normal plasma. Therefore, our method has good potential for clinical applications of TDM in ICU patients.

3.2.5. Stability. Table 3 shows the short-term, long-term, freeze-thaw (five cycles), and autosampler stabilities of TGC in plasma. Plasma samples can be stable for $24 \mathrm{~h}$ at room temperature or at $2-8^{\circ} \mathrm{C}$. The postpreparative samples were also stable in the autosampler maintained at $30^{\circ} \mathrm{C}$ for, at least, $30 \mathrm{~h}$. TGC was found to be stable for a maximum of five freeze and thaw cycles. Only three freeze and thaw cycles were reported in previous studies $[19,20]$. The long-term stability results showed that TGC was stable in human plasma for up to 130 days at $-80^{\circ} \mathrm{C}$.

3.3. Application. Severely ill patients have complex physiologies, often have liver and kidney dysfunction, and have various infections; therefore, there are large differences between their metabolisms and drug clearance. Therefore, it is necessary to detect the blood concentration to determine 

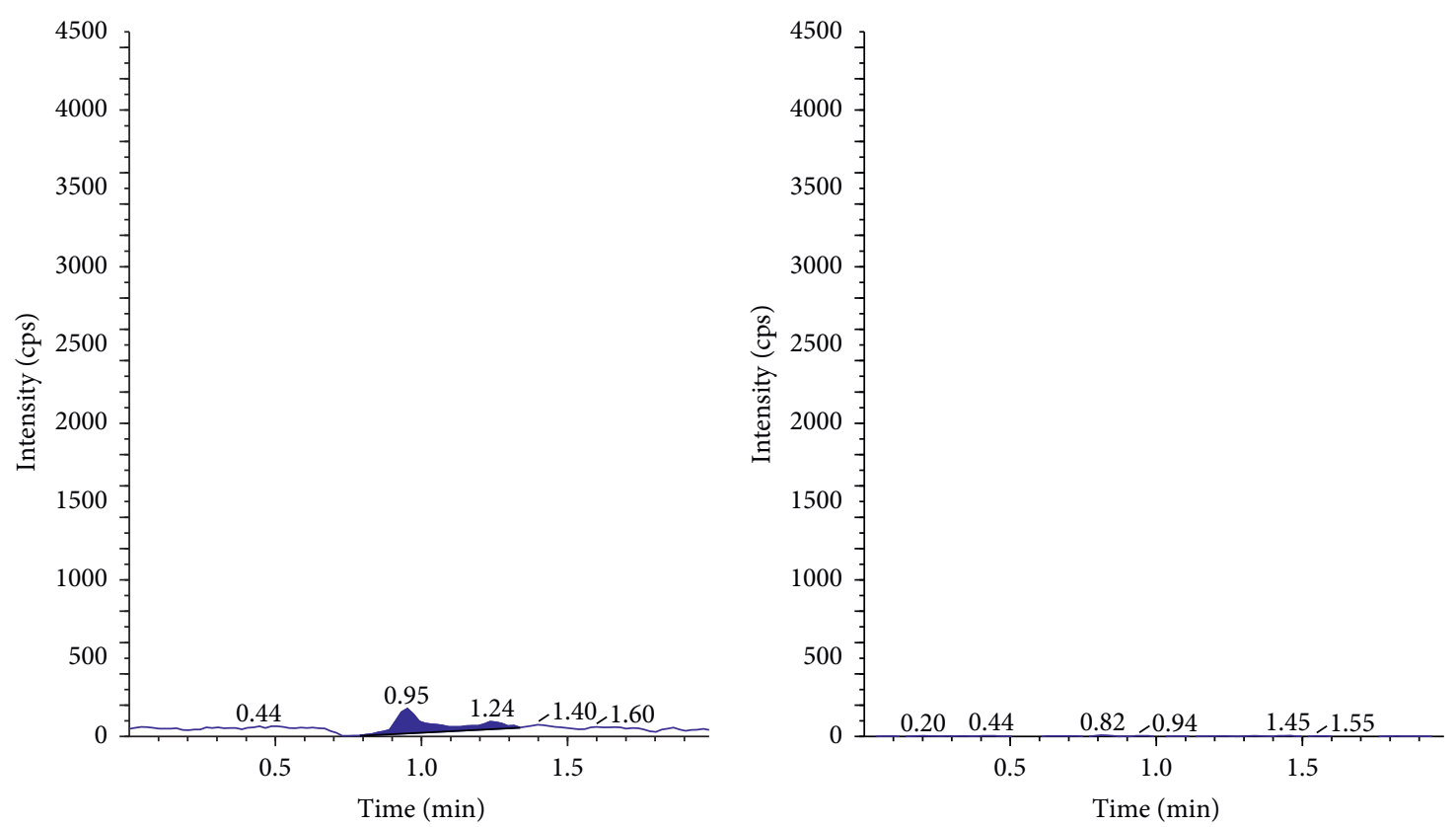

(a)
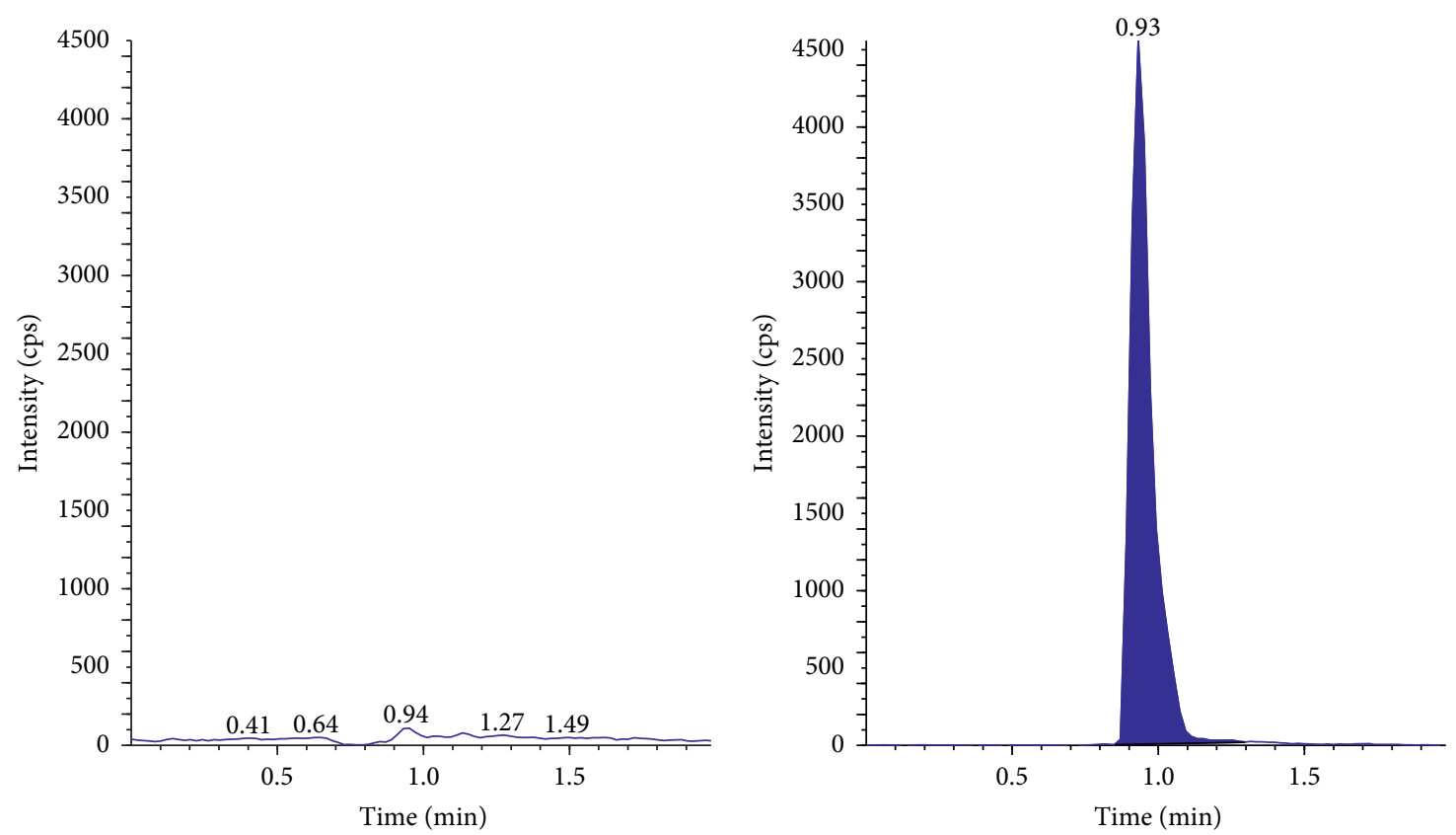

(b)

Figure 2: Continued. 

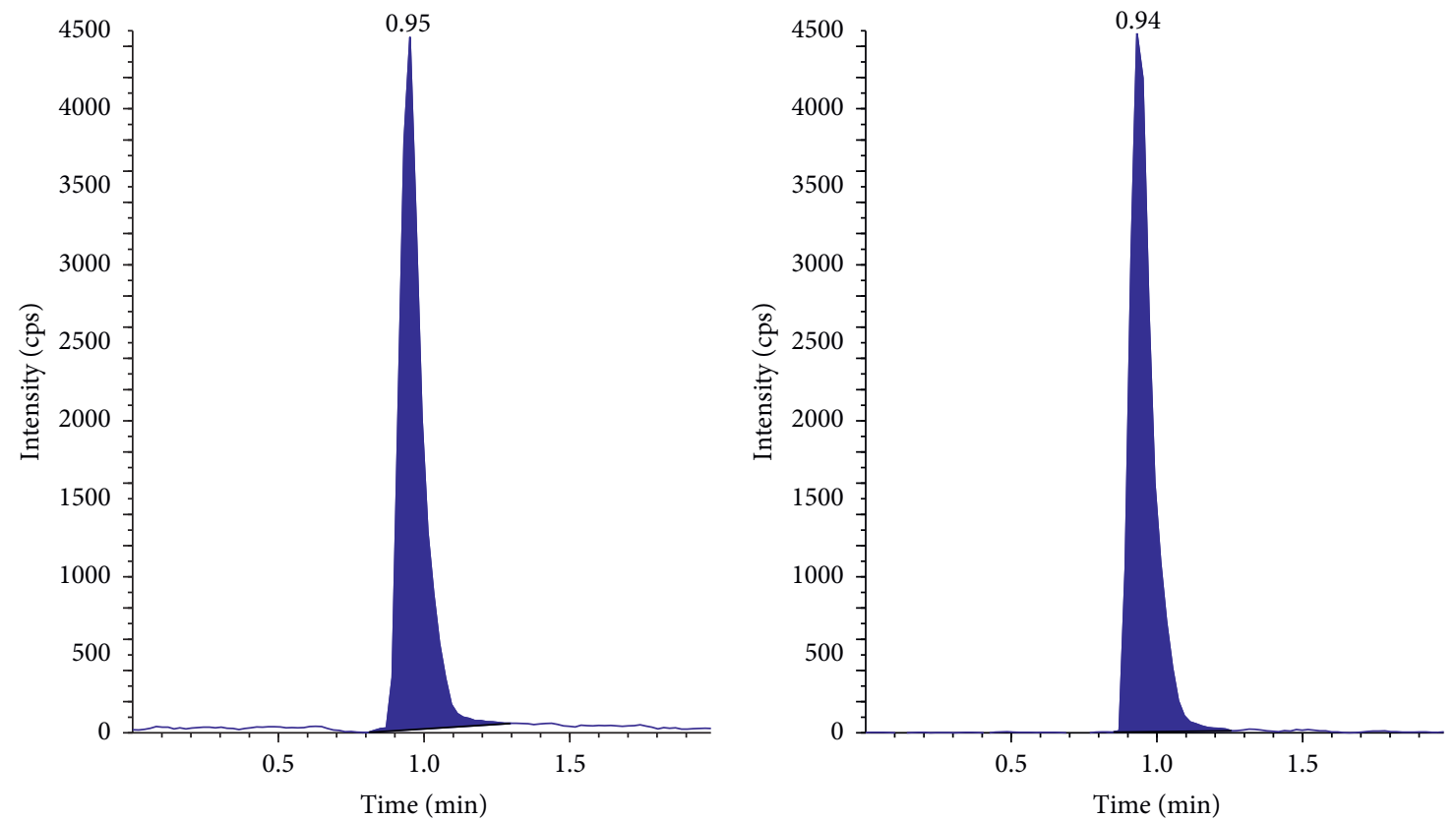

(c)
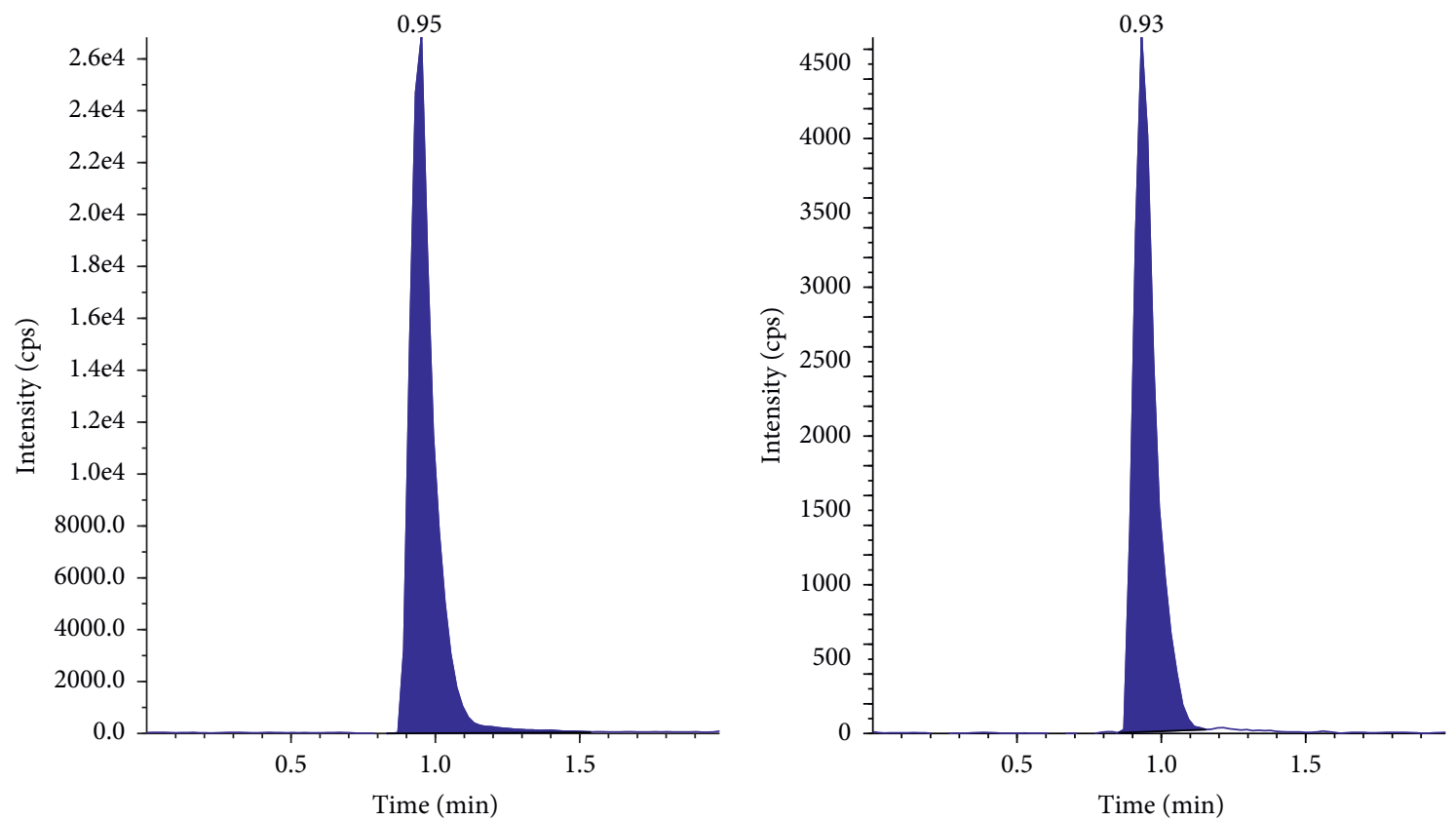

(d)

Figure 2: The chromatograms of TGC and IS in human plasma. Left is TGC, and right is IS. (a) Double-blank plasma sample; (b) blank plasma sample; (c) lower limit of quantification (LLOQ) plasma samples; and (d) a patient's plasma sample.

TABle 1: The precision and accuracy of the determination of TGC in human plasma $(n=6)$.

\begin{tabular}{|c|c|c|c|c|c|c|}
\hline \multirow[b]{2}{*}{$\begin{array}{l}\text { Nominal concentration } \\
(\mathrm{ng} / \mathrm{ml})\end{array}$} & \multicolumn{3}{|l|}{ Intrabatch $(n=6)$} & \multicolumn{3}{|l|}{ Interbatch $(n=18)$} \\
\hline & $\begin{array}{l}\text { Measured concentration }(\mathrm{ng} / \mathrm{ml} \text {, } \\
\text { mean } \pm \mathrm{SD})\end{array}$ & $\begin{array}{c}\mathrm{CV} \\
\%\end{array}$ & $\begin{array}{c}\text { Accuracy } \\
\%\end{array}$ & $\begin{array}{l}\text { Measured concentration }(\mathrm{ng} / \mathrm{ml} \text {, } \\
\text { mean } \pm \mathrm{SD})\end{array}$ & $\begin{array}{c}\mathrm{CV} \\
\%\end{array}$ & $\begin{array}{c}\text { Accuracy } \\
\%\end{array}$ \\
\hline 50 & $49 \pm 1.8$ & 3.6 & 98.6 & $47 \pm 3.3$ & 7.2 & 93.4 \\
\hline 150 & $153 \pm 2.7$ & 1.8 & 101.8 & $150 \pm 5.5$ & 3.7 & 99.7 \\
\hline 750 & $730 \pm 12.7$ & 1.7 & 97.3 & $716 \pm 30.2$ & 4.2 & 95.4 \\
\hline 2250 & $2277 \pm 48.0$ & 2.1 & 101.3 & $2206 \pm 112.8$ & 5.1 & 98.1 \\
\hline 3800 & $3565 \pm 105.4$ & 3.0 & 93.8 & $3739 \pm 214.2$ & 5.7 & 98.4 \\
\hline
\end{tabular}


TABLE 2: Matrix effect of TGC in various plasma matrices.

\begin{tabular}{|c|c|c|c|c|c|c|}
\hline \multirow{2}{*}{$\begin{array}{l}\text { Nominal } \\
\text { concentration }(\mathrm{ng} / \mathrm{ml})\end{array}$} & \multicolumn{2}{|c|}{ Normalized plasma } & \multicolumn{2}{|c|}{ Hemolyzed plasma } & \multicolumn{2}{|c|}{ Hyperlipidemic plasma } \\
\hline & $\begin{array}{c}\text { Matrix effects } \\
(\%)\end{array}$ & $\begin{array}{c}\text { IS-normalized matrix } \\
\text { effects }(\%)\end{array}$ & $\begin{array}{c}\text { Matrix } \\
\text { effects (\%) }\end{array}$ & $\begin{array}{c}\text { IS-normalized matrix } \\
\text { effects }(\%)\end{array}$ & $\begin{array}{c}\text { Matrix } \\
\text { effects (\%) }\end{array}$ & $\begin{array}{c}\text { IS-normalized matrix } \\
\text { effects }(\%)\end{array}$ \\
\hline 150 & $209 \pm 11.0$ & $103 \pm 6.9$ & $209 \pm 1.4$ & $104 \pm 5.6$ & $200 \pm 5.2$ & $95 \pm 3.2$ \\
\hline 750 & $182 \pm 1.9$ & $87 \pm 5.2$ & $193 \pm 9.3$ & $96 \pm 6.0$ & $179 \pm 1.4$ & $93 \pm 3.8$ \\
\hline 3800 & $149 \pm 1.6$ & $95 \pm 2.0$ & $152 \pm 3.4$ & $95 \pm 2.1$ & $150 \pm 1.5$ & $98 \pm 2.1$ \\
\hline CV (\%) & 14.5 & 8.5 & 13.8 & 6.0 & 12.0 & 4.0 \\
\hline
\end{tabular}

TABLE 3: Stability of TGC in human plasma under tested conditions.

\begin{tabular}{|c|c|c|c|c|}
\hline Stability & Nominal concentration $(\mathrm{ng} / \mathrm{mL})$ & Stability sample concentration $(\mathrm{ng} / \mathrm{mL})$ & Accuracy $(\%)$ & CV (\%) \\
\hline \multirow{2}{*}{ Short-term $\left(24 \mathrm{~h}, 25^{\circ} \mathrm{C}\right)$} & 150 & $168 \pm 7.0$ & 112.3 & 4.2 \\
\hline & 3800 & $3753 \pm 206.5$ & 98.8 & 5.5 \\
\hline \multirow{2}{*}{ Short-term $\left(24 \mathrm{~h}, 4^{\circ} \mathrm{C}\right)$} & 150 & $158 \pm 14.7$ & 105.4 & 9.3 \\
\hline & 3800 & $3673 \pm 212.0$ & 96.8 & 5.8 \\
\hline \multirow{2}{*}{ Freeze-thaw (five cycles, $-80-25^{\circ} \mathrm{C}$ ) } & 150 & $150 \pm 3.8$ & 100.1 & 2.5 \\
\hline & 3800 & $3993 \pm 293.6$ & 105.1 & 7.4 \\
\hline \multirow{2}{*}{ Long-term $\left(130\right.$ days, $\left.-80^{\circ} \mathrm{C}\right)$} & 150 & $156 \pm 11.1$ & 103.8 & 7.1 \\
\hline & 3800 & $3524 \pm 97.4$ & 92.7 & 2.8 \\
\hline \multirow{2}{*}{ Autosampler $\left(30 \mathrm{~h}, 30^{\circ} \mathrm{C}\right)$} & 150 & $162 \pm 10.4$ & 108.2 & 6.4 \\
\hline & 3800 & $4021 \pm 269.0$ & 105.8 & 6.7 \\
\hline
\end{tabular}

whether the conventional dose can achieve an effective treatment rate. In this study, we collected 222 samples and tested the TGC concentration in patients who received tigecycline to treat lung infections in ICUs. The peak concentration after dose $\left(C_{\max }\right.$ ) of TGC is $871.5 \pm 467.3 \mathrm{ng} / \mathrm{ml}$, the concentration within 6 hours after administration is $334.8 \pm 220.3 \mathrm{ng} / \mathrm{ml}$, and the trough concentration $\left(C_{\min }\right)$ of TGC is $260.9 \pm 190.1 \mathrm{ng} / \mathrm{ml}$.

\section{Conclusions}

The established method exhibited good specificity, precision, accuracy, and linearity in the range of 50-5000 ng/ml. Only $50 \mu \mathrm{L}$ of plasma is required, and the supernatant used for detection can be obtained by centrifuging plasma that was processed by a one-step protein precipitation method, which is quite convenient, practical, and fast. Compared with the method reported in the literature, our method has a detection time of only 2 minutes. The established method is suitable for the analysis of clinical plasma samples, including hemolyzed or hyperlipidemic samples, and was successfully applied to a PPK study of 74 ICU patients. This model can be used to estimate TGC exposure in future PK/PD studies on the efficacy and safety of TGC administered to ICU patients.

\section{Abbreviations}

$\begin{array}{ll}\text { IS: } & \text { Internal standard } \\ \text { LC-MS/ } & \text { Liquid chromatography-tandem mass } \\ \text { MS: } & \text { spectrometry } \\ \text { TGC: } & \text { Tigecycline } \\ \text { MRM: } & \text { Multireaction monitoring } \\ \text { ESI: } & \text { Electrospray ion source } \\ \text { FDA: } & \text { Food and Drug Administration }\end{array}$

AUC24: The area under the concentration-time curve from 0 to $24 \mathrm{~h}$ at steady state

MIC: Minimal inhibitory concentration

CL: $\quad$ Clearance

TDM: $\quad$ Therapeutic drug monitoring

PK/PD: Pharmacokinetic/pharmacodynamic

UV: Ultraviolet

TGC-d9: Tigecycline-d9

ICU: Intensive care unit

PPK: $\quad$ Population pharmacokinetic

HPLC: High-pressure liquid chromatography

QC: Quality control

LLOQ: Lower limit of quantification

ULOQ: Upper limit of quantification

CV\%: Coefficient variation

MF: $\quad$ Matrix factor

$C_{\text {max }}$ : The peak concentration after dose

$C_{\text {min }}$ : The trough concentration.

\section{Data Availability}

The data used to support the findings of this study are included within the article.

\section{Conflicts of Interest}

The authors declare that there are no conflicts of interest regarding the publication of this article.

\section{Authors' Contributions}

Fen Yao, Yifan Wang, and Yating Hou contributed equally to this work. FY, YTH, and CBC equally contributed to the design of the research and interpretation of the data. FY, 
YFW, and YTH contributed to the conception and design of the research, as well as interpretation of the data, and critically revised the manuscript. FY, XPW, and YFW performed the research and collected data. FY experimented and analyzed the data. All authors contributed to the acquisition and analysis of the data, drafted the manuscript, and agreed to be fully accountable for ensuring the integrity and accuracy of the work. All authors read and approved the final manuscript.

\section{Acknowledgments}

The authors would like to thank all the doctors, nurses, technicians, and patients involved in the ICU for their dedication to the study. This work was supported by the National Natural Science Foundation of China (Grant no.81671963); the Guangzhou Science and Technology Program (Grant no.201803010058); the Major Program of Summit Project, Guangdong Province High-level Hospital Construction Project of Guangdong Provincial People's Hospital, Guangdong Academy of Medical Sciences (Grant no.DFJH2020028); and the Guangdong Provincial Hospital Pharmaceutical Research Fund (ChiaTai Tianqing) of Guangdong Pharmaceutical Association (Grant no. 2018A04). This study was also supported by the High-level Hospital Construction Research Project of Maoming People’s Hospital.

\section{References}

[1] K. Brust, A. Evans, and R. Plemmons, "Tigecycline in treatment of multidrug-resistant gram-negative bacillus urinary tract infections: a systematic review," Journal of Antimicrobial Chemotherapy, vol. 69, no. 10, pp. 2606-2610, 2014.

[2] Z. Y. Lu, N. Xu, B. L. He et al., "Inhibition of autophagy enhances the selective anti-cancer activity of tigecycline to overcome drug resistance in the treatment of chronic myeloid leukemia," Journal of Experimental \& Clinical Cancer Research, vol. 36, 2017.

[3] L. Zha, L. Pan, J. Guo, N. French, E. V. Villanueva, and B. Tefsen, "Effectiveness and safety of high dose tigecycline for the treatment of severe infections: a systematic review and meta-analysis," Advances in Therapy, vol. 37, no. 3, pp. 1049-1064, 2020.

[4] G. De Pascale, L. Lisi, G. M. P. Ciotti et al., "Pharmacokinetics of high-dose tigecycline in critically ill patients with severe infections," Annals of Intensive Care, vol. 10, no. 1, p. 94, 2020.

[5] R. Alraish, S. G. Wicha, O. R. Frey et al., "Pharmacokinetics of tigecycline in critically ill patients with liver failure defined by maximal liver function capacity test (LiMAx)," Annals of Intensive Care, vol. 10, no. 1, p. 106, 2020.

[6] FDA, Tigecycline for Injection, for Intravenous Use Label, FDA, Silver Spring, MD, USA, 2005.

[7] M. Yadav, R. Rao, H. Kurani et al., "Validated ultra-performance liquid chromatography tandem mass spectrometry method for the determination of pramipexole in human plasma," Journal of Chromatographic Science, vol. 48, no. 10, pp. 811-818, 2010.

[8] X. Ling, Y. Q. Xiang, Q. F. Tang, Z. Jin, F. L. Chen, and X. M. Tan, "Soybean milk inhibits absorption and intestinal transmembrane transport of gegen in rats," Evidence-Based
Complementary and Alternative Medicine, vol. 2017, Article ID 7146813, 11 pages, 2017.

[9] J.-y. Chen, M. Yi, S.-l. Yao, and X.-p. Zhang, "Propofol targetcontrolled infusion modeling in rabbits: pharmacokinetic and pharmacodynamic analysis," Journal of Huazhong University of Science and Technology (Medical Sciences), vol. 36, no. 3, pp. 428-433, 2016.

[10] C. M. Rubino, S. M. Bhavnani, A. Forrest et al., "Pharmacokinetics-pharmacodynamics of tigecycline in patients with community-acquired pneumonia," Antimicrobial Agents and Chemotherapy, vol. 56, no. 1, pp. 130-136, 2012.

[11] A. Broeker, S. G. Wicha, C. Dorn et al., "Tigecycline in critically ill patients on continuous renal replacement therapy: a population pharmacokinetic study," Critical Care, vol. 22, no. 1, p. 341, 2018.

[12] J. Mohri, C. Katada, M. Ueda et al., "Predisposing factors for chemotherapy-induced nephrotoxicity in patients with advanced esophageal cancer who received combination chemotherapy with docetaxel, cisplatin, and 5-fluorouracil," Journal of Translational Internal Medicine, vol. 6, no. 1, pp. 32-37, 2018.

[13] C. M. Rubino, A. Forrest, S. M. Bhavnani et al., "Tigecycline population pharmacokinetics in patients with community- or hospital-acquired pneumonia," Antimicrobial Agents and Chemotherapy, vol. 54, no. 12, pp. 5180-5186, 2010.

[14] Y. Deng, R. Chi, S. Chen et al., "Evaluation of clinically available renal biomarkers in critically ill adults: a prospective multicenter observational study," Critical Care, vol. 21, no. 1, p. 46, 2017.

[15] Y. Deng, L. Wang, Y. Hou et al., "The influence of glycemic status on the performance of cystatin $\mathrm{C}$ for acute kidney injury detection in the critically ill," Renal Failure, vol. 41, no. 1, pp. 139-149, 2019.

[16] Y. Deng, J. Yuan, R. Chi et al., “The incidence, risk factors and outcomes of postoperative acute kidney injury in neurosurgical critically ill patients," Scientific Reports, vol. 7, no. 1, p. 4245, 2017.

[17] Y. Wu, W. Peng, R. Wei et al., "Rat mRNA expression profiles associated with inhibition of ischemic acute kidney injury by losartan," Bioscience Reports, vol. 39, no. 4, 2019.

[18] D. Zhang, L. Gao, H. Ye et al., "Impact of thyroid function on cystatin $\mathrm{C}$ in detecting acute kidney injury: a prospective, observational study," BMC Nephrology, vol. 20, no. 1, p. 41, 2019.

[19] R. Shao, X. Li, Y. Hu, J. Chen, H. Lou, and H. Dai, "Determination of tigecycline in human plasma by LC-MS/MS and its application to population pharmacokinetics study in Chinese patients with hospital-acquired pneumonia," Biomed Chromatogr, vol. 32, no. 2, 2018.

[20] S. Mei, X. Luo, X. Li et al., "Development and validation of an LC-MS/MS method for the determination of tigecycline in human plasma and cerebrospinal fluid and its application to a pharmacokinetic study," Biomedical Chromatography, vol. 30, no. 12, pp. 1992-2002, 2016.

[21] A. D'Avolio, E. Peila, M. Simiele et al., "Ultra performance liquid chromatography PDA method for determination of tigecycline in human plasma," Therapeutic Drug Monitoring, vol. 35, no. 6, pp. 853-858, 2013.

[22] J. Xie, T. Wang, X. Wang et al., "Quantitative analysis and pharmacokinetics study of tigecycline in human serum using a validated sensitive liquid chromatography with tandem mass spectrometry method," Journal of Separation Science, vol. 37, no. 12, pp. 1396-1403, 2014.

[23] F. Qiu, B. Su, Z. Li et al., "New serum biomarker identification and analysis by mass spectrometry in cervical precancerous 
lesion and acute cervicitis in South China," Cancer Management and Research, vol. 11, pp. 6151-6162, 2019.

[24] Z. Zhang, K. Li, M. Yan et al., "Metabolomics profiling of cleidocranial dysplasia," Clinical Oral Investigations, vol. 23, no. 3, pp. 1031-1040, 2019.

[25] L. L. Pan, Q. H. Sun, G. R. Liu, and J. Y. Guo, "Urinary metabolomics study of the intervention effect of hypoglycemic decoction on type 2 diabetes mellitus rats model," Evidence-Based Complementary and Alternative Medicine, vol. 2019, Article ID 1394641, 17 pages, 2019.

[26] B. Ingelse, B. Barroso, N. Gray et al., "European bioanalysis forum: recommendation on dealing with hemolyzed and hyperlipidemic matrices," Bioanalysis, vol. 6, no. 23, pp. 3113-3120, 2014.

[27] E.-R. Bérubé, M.-P. Taillon, M. Furtado, and F. Garofolo, "Impact of sample hemolysis on drug stability in regulated bioanalysis," Bioanalysis, vol. 3, no. 18, pp. 2097-2105, 2011.

[28] W. Ding, T. Wang, L. H. Liu et al., "Phenotypic modulation of corpus cavernosum smooth muscle cells in a hyperlipidemic rat model," International Journal of Clinical and Experimental Pathology, vol. 10, no. 3, pp. 3052-3060, 2017.

[29] Y. X. Chen, X. Q. Wang, Y. Fu et al., "Pivotal role of inflammation in vascular endothelial dysfunction of hyperlipidemic rabbit and effects by atorvastatin," International Journal of Cardiology, vol. 146, no. 2, pp. 140-144, 2011. 